\title{
Opacidade contra-alimentadora no PE: análise por Marcação Comparativa
}

\author{
Counter-feeding Opacity in European Portuguese: a \\ Comparative Markedness analysis
}

Gisela Collinschonn

Universidade Federal do Rio Grande do Sul (UFRGS)

\begin{abstract}
Recent generative phonology is witnessing a debate between several proposals concerning the solution of the opacity problem. "Opacity" refers to generalizations that are not superficially detectable - which are explained in derivational phonology by applying ordered application of rules. In Optimality Theory, the opacity issue attracts special interest, since this theory abandons rules altogether, such as intermediate representations and derivational stages between input and output. As for the responses to the opacity issue, some propose a return in OT to derivational levels, similar to the components of Lexical Phonology (KIPARSKY, 2000; RUBACH, 2000; ITÔ; MESTER, 2003). These solutions give up full parallelism, since evaluation of well-formedness is realized in a staged form. Other solutions maintain parallelism and appeal to other resources of the theory to account for the issue, like conjoined constraints (KIRCHNER, 1996; McCARTHY, 1999), specializations of correspondence constraints (Sympathy Theory) or markedness constraints (Comparative Markedness Theory). Some of these alternative solutions are, in some way, derivational or almost derivational (MCARTHY, 2006, p.5), because they appeal to an intermediate form to influence the output. In this paper, we discuss two phenomena from European Portuguese in which interaction is opaque. The analysis is restricted to counter-feeding opacity and focalizes an account according to Comparative Markedness (McCARTHY, 2002a). Additionally, we discuss some of the implications of this account, relative to the Richness of the Base postulate, and some predictions concerning the possibility of interaction with other processes.
\end{abstract}




\section{Keywords}

Optimality Theory, Opacity, Comparative Markedness

\section{Resumo}

No quadro atual da literatura fonológica gerativa, há um amplo debate entre as várias propostas de solução para o problema da opacidade. O termo "opacidade" refere-se a generalizações fonológicas que não são superficialmente detectáveis - que a fonologia derivacional explica pela aplicação ordenada de regras. Na Teoria da Otimidade, a questão da opacidade torna-se especialmente relevante, já que essa teoria abre mão de regras, bem como de representações intermediárias e de estágios derivacionais entre input e output. Entre as respostas ao problema da opacidade, algumas propõem o retorno a níveis semelhantes aos componentes da Fonologia Lexical (KIPARSKY, 2000; RUBACH, 2000; ITÔ; MESTER,2003). Essas soluções abrem mão do paralelismo pleno, isto é, admitem estágios de avaliação, embora a avaliação das formas num determinado estágio seja feita em paralelo. Outras soluções mantêm o paralelismo, e lançam mão de outros aspectos da teoria para dar conta do problema, tais como restrições conjuntas (KIRCHNER, 1996; McCARTHY, 1999), ou apelam para especializações das restrições de correspondência (Teoria da Simpatia) ou de marcação (Teoria da Marcação Comparativa). Algumas das alternativas de solução apresentadas nos últimos anos são, de certa forma, derivacionais ou quase derivacionais (McCARTHY, 2006a, p.5), porque apelam para uma forma intermediária para influenciar o output. Neste texto, abordamos dois fenômenos do português europeu cuja interação é opaca. A análise restringe-se ao tipo de opacidade que surge quando dois processos se aplicam de acordo com o ordenamento chamado contra-alimentador e focaliza uma das alternativas de abordagem dessa opacidade, a da Marcação Comparativa (McCARTHY, 2002a). Concluímos a apresentação discutindo algumas implicações dessa abordagem, relacionadas ao postulado da riqueza da base e a predições a respeito das possibilidades de interação com outros processos.

\section{Palavras-chave}

Teoria da Otimidade, Opacidade, Marcação Comparativa 


\section{Introdução}

$\mathrm{E}$

ste texto constitui-se de anotações sobre o tratamento da opacidade em

Teoria da Otimidade (TO) elaboradas a partir de uma disciplina

ministrada no Programa de Pós-Graduação em Letras da UFRGS. Nele, discutimos um caso da opacidade observado no português europeu e as dificuldades que essa opacidade traz para a Teoria da Otimidade. Devido à atualidade do tema no âmbito das discussões teóricas em fonologia, acreditamos que as reflexões aqui apresentadas possam ser úteis a outros pesquisadores.

O termo "opacidade" refere-se a generalizações fonológicas que não são superficialmente detectáveis - que a fonologia derivacional explica pela aplicação ordenada de regras. Na Teoria da Otimidade, a questão da opacidade torna-se especialmente relevante, já que essa teoria abre mão de regras, bem como de representações intermediárias e de estágios derivacionais entre input e output. Algumas das alternativas de solução apresentadas nos últimos anos são, de certa forma, derivacionais ou quase derivacionais, como o próprio McCarthy reconhece em texto recente (McCARTHY, 2006a, p.5), porque apelam para uma forma intermediária para influenciar o output. A nossa análise focalizará uma dessas alternativas, a Marcação Comparativa (McCARTHY, 2002a).

O conceito de opacidade e, relacionado a este, de relações de alimentação e sangramento entre regras vêm do trabalho de Kiparsky $(1968,1971)$ sobre mudança lingüística. A opacidade surge de determinados tipos de ordenamento entre regras. Considerem-se duas regras, A e B, sendo que A está ordenada antes de B. A interação é:

(1) a. alimentadora se A cria contextos em que B pode se aplicar;

b. sangradora se A desfaz contextos em que B poderia se aplicar;

c. contra-alimentadora se B alimentaria A caso a ordem fosse inversa;

d. contra-sangradora se B sangraria A caso a ordem fosse inversa. 
Para ilustrarmos, vejamos alguns casos de interações entre regras do português brasileiro. A alimenta $\mathrm{B}$ - um exemplo é a elevação da vogal átona, que cria contextos para a palatalização das oclusivas dentais: lejte > lejti > lejt $\int \mathrm{i}$ (BISOL, 1991; ABAURRE; PAGOTTO, 2002). Se a palatalização fosse uma regra do léxico, podemos imaginar que ela se aplicaria antes da elevação da vogal átona, ou seja, não estaria mais disponível quando as vogais fossem elevadas. Se assim fosse, a regra de elevação contra-alimentaria a de palatalização, como pode ser visto na parte direita do quadro seguinte (exemplo 2).

(2)

$\begin{array}{llll} & \text { Alimentação } & & \text { Contra-alimentação } \\ \text { RSubj } & \text { /lejte/ } & \text { RSubj } & \text { /lejte/ } \\ \text { elevação } & \text { lejti } & \text { palatalização } & - \\ \text { palatalização } & \text { lejt } \int \mathrm{i} & \text { elevação } & \text { lejti } \\ \text { RSup } & {\left[\text { lejt } \int \mathrm{I}\right]} & \text { RSup } & *[\text { lejtr] }\end{array}$

Há outros casos de alimentação no português brasileiro. Por exemplo, Vieira (2002) constata que a elevação da átona final é bloqueada quando for seguida de coda soante, mas, quando essa consoante for apagada, como em home, garage, revolve, há uma tendência forte de a vogal elevar-se para [i]. Dessa forma, o apagamento da consoante final cria o contexto para elevação da átona final. Se esse apagamento ocorresse somente depois que a elevação de átona final deixou de ser ativa, esperaríamos formas como [õme], mas não [õmi], ou seja, o apagamento da consoante contra-alimentaria a elevação da vogal.

Nossa análise será restrita a casos de contra-alimentação, isto é, o caso inverso da alimentação. Os dados com que vamos trabalhar neste texto vêm de uma situação de contra-alimentação observada no português europeu, entre a centralização da vogal/e/ antes de segmento palatal, como em ab[e]lha e l[e] $]$, e a palatalização da fricativa pós-vocálica. Os dois fenômenos são ilustrados com alguns exemplos a seguir (conforme VIGÁRIO, 2003, p.79 e MATEUS; D'ANDRADE, 2000, p.12):

$$
\begin{aligned}
& \text { a. centralização de /e/ } \\
& \text { lenha [en] cereja [e3] } \\
& \text { telha }[\mathrm{e} K] \text { fecho }[\mathrm{e} f]
\end{aligned}
$$

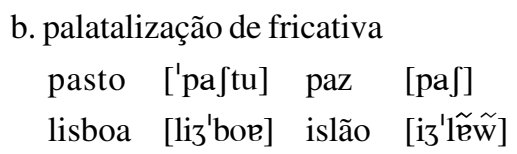

A regra de centralização não é alimentada pela regra de palatalização, o que evidenciam os exemplos (4) e (5): 


$$
\begin{array}{ll}
\text { incesto }\left[\mathrm{e} \int\right] / *\left[\mathrm{e} \int\right] & \text { resma }\left[\mathrm{e}_{3}\right] / *\left[\mathrm{e}_{3}\right] \\
\text { vespa }\left[\mathrm{e} \int\right] / *\left[\mathrm{e} \int\right] & \text { mesmo }\left[\mathrm{e}_{3}\right] / *\left[\mathrm{e}_{3}\right] \\
\text { texto }\left[\mathrm{e} \int\right] / *\left[\mathrm{e} \int\right] & \text { lesma }\left[\mathrm{e}_{3}\right] / *\left[\mathrm{e}_{3}\right]
\end{array}
$$

Aqui, representamos no lado esquerdo o ordenamento correto, contraalimentador. No lado direito, mostramos que, se o ordenamento fosse inverso,

\begin{tabular}{|c|c|c|c|}
\hline \multirow{4}{*}{$\begin{array}{l}\text { RSubj } \\
\text { centralização } \\
\text { palatalização }\end{array}$} & \multicolumn{2}{|c|}{ Contra-alimentação } & Alimentação \\
\hline & /veSpa/ & RSubj & /veSpa/ \\
\hline & 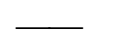 & palatalização & ve $\int p a$ \\
\hline & ve $\int p a$ & centralização & ve $\int p a$ \\
\hline RSup & [vepe] & RSup & $*\left[\mathrm{ve} \int \mathrm{pe}\right]$ \\
\hline
\end{tabular}
seria alimentador.

(5)

Se olharmos exclusivamente para a forma de superfície, [ve $\left.\int p e\right]$, não sabemos por que a centralização não se aplicou. Na verdade, é o ordenamento contra-alimentador o grande responsável pela não-aplicação da regra, pois, no estágio derivacional em que a centralização está ativa, o contexto de consoante palatal ainda não existe na forma da palavra "vespa".

Opacidade caracteriza uma situação em que uma regra não parece ser válida na representação superficial. Numa relação de contra-alimentação, a regra A não se aplica, embora a representação superficial apresente o contexto para sua aplicação. Isso porque esse contexto somente foi produzido depois que a regra A deixou de ser ativa. Numa relação de contra-sangramento, a regra $\mathrm{A}$ se aplica, mas alterações posteriores modificam as condições que permitiram a sua aplicação. Assim, a regra parece ter se aplicado sem ter o contexto para tal. Em ambos os casos, uma generalização não é válida na superfície, o que caracteriza a opacidade.

Na seqüência, apresentamos com mais detalhe os fenômenos do português europeu discutidos aqui. Em seguida, mostramos os problemas que se apresentam para uma análise por restrições e propomos uma alternativa através da Teoria da Marcação Comparativa. Finalmente, discutimos algumas questões relativas a essa alternativa para abordagem da opacidade em TO, especialmente no que concerne a predições que essa abordagem faz que são falseadas pelos fenômenos aqui analisados. 


\section{Os dados}

Nesta sessão, os fenômenos do português europeu são apresentados de acordo com sua análise pela perspectiva derivacional. Os textos nos quais nos apoiamos principalmente são de Mateus et al. (1990), Mateus e d'Andrade (2000) e Vigário (2003).

Como vimos, a centralização atinge a vogal /e/ quando seguida de segmento palatal (exemplos conforme MATEUS et al., 1990, p.326).

(6) lenha ['lene]

cereja [sə'rвze] [sә'rвjзe $]$

telha ['teאe $]$

fecho $\quad\left[\right.$ 'fe $\left.\int \mathrm{u}\right] \sim\left[\right.$ 'frj $\left.\int \mathrm{u}\right]$

areia [e'reje]

Segundo Mateus e d'Andrade (2000, p.17), a centralização é própria do dialeto de Lisboa e arredores. Ao que parece, o fenômeno se originou em Lisboa e se estendeu à pronúncia culta de todo o Portugal. Em alguns dialetos, a regra atinge também a vogal subjacente $/ \varepsilon /$.

Mateus et al.(1990, p. 327) expressam o fenômeno através de duas regras distintas: a centralização antes de glide e a centralização antes de consoante palatal. Entretanto, a regra pode ser formulada de forma a unificar os dois contextos, como propõe d'Andrade 1994, apud Vigário, 2003):

(7)

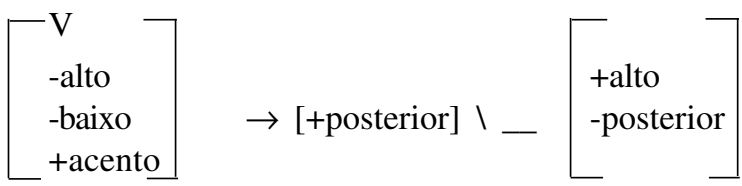

Como vemos, pela formalização da regra, esse fenômeno é entendido como um caso de dissimilação. Vigário (2003) adota essa formulação, mas considera que há duas regras, uma em que a centralização ocorre apenas antes de glide e outra em que ocorre antes de qualquer segmento palatal. Esta segunda regra é formalizada semelhantemente à regra acima, com a diferença de que a autora insere uma condição: a de que os segmentos adjacentes sejam heterossilábicos. Com essa condição, excluem-se da aplicação casos como os em (4), conforme Vigário (2003, p. 80). A autora traz como argumento para essa divisão em duas da regra de centralização o fato de que a centralização tautossilábica não admite exceções, diferentemente da centralização heterossilábica (ex.: mexo). ${ }^{1}$ 
(8)

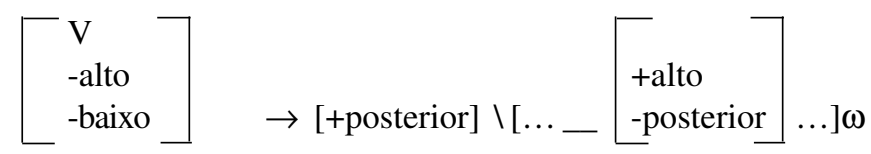

(quando os dois segmentos adjacentes forem heterossilábicos)

(conforme VIGÁRIO, 2003,p. 81, n.104)

Entretanto tal condição não nos parece necessária. Em primeiro lugar, parece um tanto estranha, pois estabelece que uma consoante interage com uma vogal desde que não haja uma relação local estreita entre elas, quando o esperado seria o contrário, num caso dissimilatório como este. Em segundo lugar, o ordenamento contra-alimentador entre palatalização e centralização, como justificado a seguir, dá conta da não-aplicação nos casos em (4). Portanto, se consideramos válido esse ordenamento, a condição torna-se desnecessária.

Finalmente, no que se refere à divisão da centralização em duas regras distintas, consideramos que faltam outros elementos para sustentá-la; não há, por exemplo, evidência de ordenamento distinto entre as duas regras. Como tanto a descrição estrutural (incluindo o contexto) quanto a mudança estrutural são similares, acreditamos que seja acertado considerá-las uma só regra. Portanto, adotamos, para fins da presente argumentação, a regra como proposta em (7).

Argumentos para o status lexical da regra são apresentados em Vigário (2003, p.82): (a) a regra possui exceções: mexo [e $\left.\int\right] / *\left[\mathrm{e} \int\right]$ e rejo [e3]/*[[3] comparado com protejo *[e3]/ [e3]; e (b) a inserção pós-lexical de um glide palatal não alimenta a regra: lêem [lejẽj]]/ *[lejẽj]. Vigário (2003) reconhece que a regra não é preservadora de estrutura, pois cria um segmento não-presente no inventário fonológico segmental. Entretanto, a autora considera que a obediência à preservação de estrutura não caracteriza todas as regras do componente lexical, mas apenas aquelas que fazem parte do estrato cíclico, posição com a qual concordamos.

Vemos, portanto, a partir da análise de Vigário (2003), que a centralização deve ser lexical, entre outros argumentos, porque é contra-alimentada por regras que têm aplicação no pós-léxico. No que se refere à palatalização, Vigário (2003, p.80) descarta a interpretação de relação contra-alimentadora proposta em d'Andrade (1994). ${ }^{2}$ Entretanto, no nosso entender, a palatalização da fricativa pós-vocálica é claramente pós-lexical, como demonstra o fato de não se aplicar quando ocorre a ressilabação entre palavras: maus amigos [máwzemígu]], duas almas [dúezałme]] (de MATEUS e D'ANDRADE, 2000). ${ }^{3}$ Com essas considerações, a interação 
contra-alimentadora entre as duas regras é consequiência do fato de o componente pós-lexical ser ordenado depois do componente lexical, ou seja, o ordenamento contra-alimentador não precisa ser estipulado independentemente de outras propriedades das duas regras.

\section{Implicações da opacidade para a Teoria da Otimidade}

Como vimos, o termo "opacidade" refere-se a generalizações fonológicas que não são superficialmente detectáveis - que a fonologia derivacional explica pela aplicação de regras intermediárias. ${ }^{4}$

A opacidade é um problema para a TO clássica porque essa teoria prevê que não há estágios intermediários. Todas as restrições, quando estiverem ativas,

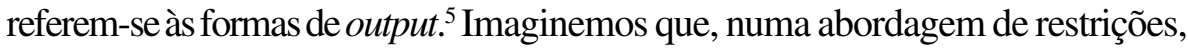
tenhamos as restrições *e $\int$, que responde pela centralização, e * $[+a n t] \sigma$, (conforme LEE, 2002) que responde pela palatalização.

(9) $*$ e $\int$ - é proibida a vogal [e] antes de fricativa [+coronal, -anterior ${ }^{6}$

*[+ant $] \sigma$ - é proibida obstruinte [+anterior] em final de sílaba

(conforme LEE, 2002) ${ }^{7}$

Elas dominam, respectivamente, as restrições de fidelidade $\operatorname{Ident}_{[\text {post] }} \mathrm{e}$ Ident $_{\text {[ant] }}$ como demonstramos com a análise das palavras "fecho" e "pasto". 8

(10) Tableau $* \mathrm{e} \int>>$ Ident $_{\text {[post] }}$

\begin{tabular}{|c|c|c|}
\hline$/ \mathrm{fe} \int \mathrm{o} / *$ & ef & Ident $_{[\text {post] }}$ \\
\hline $\mathrm{a} .\left[\mathrm{fe} \int \mathrm{u}\right]$ & $*$ & \\
\hline$\approx \mathrm{b} .\left[\mathrm{fe} \int \mathrm{u}\right]$ & & $*$ \\
\hline
\end{tabular}

(11)

\begin{tabular}{|c|l|l|}
\multicolumn{1}{ll}{ Tableau: } & $*[+\mathrm{ant}] \sigma_{>>}$ & Ident $_{\text {[ant] }}$ \\
\hline /pasto/ & $*[+\mathrm{ant}] \sigma$ & Ident $_{[\mathrm{ant}]}$ \\
\hline a.[pastu] & $*$ & \\
\hline$\approx$ b.[paStu] & & $*$ \\
\hline
\end{tabular}

Vejamos, então, as restrições reunidas na análise de "vespa". Sabemos que [ve $\left.\int \mathrm{pa}\right]$ é melhor do que [vespa], portanto $*[\operatorname{tant}] \sigma>>* \int$. 
(12) Tableau: $*[+$ ant $] \sigma>>* e \int>>$ Ident $_{\text {[post] }}$, Ident $_{\text {[ant] }}$

\begin{tabular}{|c|c|c|c|c|}
\hline /vespa / & $*[+$ ant $] \sigma$ & $*_{\mathrm{e}} \int$ & Ident $_{\text {[post] }}$ & Ident $_{\text {[ant] }}$ \\
\hline a.[vespe] & $*$ & & & \\
\hline $\begin{array}{ll}G & \text { b. [ve }[\mathrm{p}]\end{array}$ & & * & & $*$ \\
\hline
\end{tabular}

No entanto, [ve $\int \mathrm{pa}$ ] não é o vencedor quando se considera o candidato [ve $\int \mathrm{pa}$ ], no qual se observam tanto centralização quanto palatalização.

(13)

\begin{tabular}{|c|l|l|l|l|}
\hline$/$ vespa / & $*[+\mathrm{ant}] \sigma$ & $* \mathrm{e} \int$ & Ident $_{\text {[post] }}$ & Ident $_{\text {[ant] }}$ \\
\hline a.[vespe] & $*$ & & & \\
\hline b.[ve $\left.\int \mathrm{pe}\right]$ & & $*$ & & $*$ \\
\hline$\sigma \mathrm{c} .\left[\mathrm{ve} \int \mathrm{pe}\right]$ & & & $*$ & $*$ \\
\hline
\end{tabular}

O que temos é que a forma atestada viola, sem nenhum motivo aparente, uma generalização que tem um papel ativo na língua e, assim, um candidato que não viola a restrição sai-se melhor na disputa. Em TO, espera-se, em caso de conflito entre restrições, que as mais altamente ranqueadas obriguem os candidatos ótimos a violações das restrições mais baixas no ranqueamento. $\mathrm{O}$ problema que se apresenta no caso anterior é que, na forma atestada [vespa], há uma violação à restrição *e $\int$, mas ela parece gratuita, pois não há outra restrição no ranking que obrigue a essa violação.

A análise exposta é apenas esquemática, o que pode levar ao entendimento de que uma reformulação no conjunto das restrições possa contornar o problema. Entretanto, esse tipo de opacidade, que a fonologia derivacional explica com contra-alimentação entre regras, exige uma solução complexa na TO.

McCarthy (1999) explica a noção de opacidade relacionada ao ordenamento contra-alimentador da seguinte forma:

Formas deixam de sofrer um processo embora, na superfície, pareçam enquadrar-se na descrição estrutural adequada para sua aplicação. Uma generalização é não-verdadeira na superfície. $\mathrm{O}$ efeito é de subaplicação de uma regra.

Entre as respostas ao problema, várias propõem o retorno a níveis, semelhantes aos componentes lexical e pós-lexical da Fonologia Lexical (ver 
KAGER, 1999, cap. 9; KIPARKSY, 2000; RUBACH, 2000 e ITÔ; MESTER, 2003). Essas soluções abrem mão do paralelismo pleno, isto é, há estágios de avaliação, embora em cada estágio a avaliação seja feita em paralelo. Outras soluções mantêm o paralelismo, e lançam mão de outros aspectos da teoria, tais como restrições conjuntas (KIRCHNER, 1996, McCARTHY, 1999), ou apelam para especializações das restrições de correspondência (Teoria da Simpatia) ou de marcação (Teoria da Marcação Comparativa). Na próxima seção, apresentamos brevemente a Teoria da Marcação Comparativa (McCARTHY, 2002a).

\section{Marcação comparativa - a marcação desdobrada}

A proposta da marcação comparativa modifica um dos enunciados básicos da Teoria da Otimidade: o de que as restrições de marcação não têm acesso ao input. Vejamos como isso funciona com as palavras fecho e vespa.

\begin{tabular}{|c|c|c|}
\hline$/ \mathrm{fe} \int \mathrm{o} /$ & $* \mathrm{e} \int$ & Ident $_{\text {[post] }}$ \\
\hline a.[fe $\left.\int \mathrm{u}\right]$ & $*$ & \\
\hline$\sigma$ b.[freu] & & $*$ \\
\hline
\end{tabular}

No tableau acima, vemos que o candidato a. viola a restrição de marcação * e e esta violação já está presente no input. Por outro lado, no caso de vespa, a violação a *e $\int$ não está presente no input.

\begin{tabular}{|c|c|c|}
\hline$/$ vespa/ & $* \mathrm{e} \int$ & Ident $_{\text {[post] }}$ \\
\hline a.[ve $\left.\int \mathrm{pe}\right]$ & $*$ & \\
\hline b.[ve $\left.\int \mathrm{pe}\right]$ & & $*$ \\
\hline
\end{tabular}

Até essa proposta, as restrições de marcação, diferentemente das restrições de fidelidade, não podiam olhar para o input. McCarthy (2002a) propõe uma modificação nesse sentido: as restrições de marcação podem ter acesso ao que está no input. Para garantir a análise da opacidade, as restrições de marcação são divididas em dois tipos, as de marcações velhas, que são violadas apenas se a estrutura marcada estiver também no input e as de marcações novas, que são violadas apenas se a estrutura marcada não estiver no input.

$\mathrm{O}$ acesso das restrições de marcação ao input é intermediado por um dos candidatos a output. Segundo McCarthy (2002a), sempre existe, entre o conjunto 
de candidatos a output, um que é completamente fiel ao input (CPF). As restrições de marcação podem olhar para esse candidato e conferir se as violações de marcação já se encontram nele. Se esse for o caso, então o candidato a output será penalizado pela restrição de marcação velha, mas não pela nova.

No tableau seguinte, destacamos o CPF dos outros candidatos. A restrição ${ }_{\mathrm{v}}{ }^{*} \mathrm{e} \int$ verifica se os candidatos possuem uma violação idêntica à que está no input, caso do candidato a, que, por esse motivo, recebe marca de violação.

(16)

\begin{tabular}{|c|l|l|}
\hline$/ \mathrm{fe} \int \mathrm{o} /$ & $\mathrm{v} * \mathrm{e}$ & Ident $_{\text {[post] }}$ \\
\hline $\mathrm{CPF}[\mathrm{feSo}]$ & $*$ & \\
\hline a.[fe]u] & $*$ & \\
\hline b.[fe]u] & & $*$ \\
\hline
\end{tabular}

O esquema de ranking, para explicar casos como opacidade contraalimentadora, é o seguinte: ${ }_{\mathrm{v}} \mathrm{M}>>\mathrm{F}>>{ }_{\mathrm{N}} \mathrm{M}$. No tableau a seguir, ${ }_{\mathrm{v}} \mathrm{e} \int \mathrm{d}$ domina Ident $_{\text {[post] }}$, a qual, por sua vez, domina ${ }_{\mathrm{N}}{ }^{\mathrm{e}} \int\left(_{\mathrm{N}} \mathrm{e}_{\mathrm{V}}\right.$ indicam 'novo' e 'velho' respectivamente).

\begin{tabular}{|c|c|c|c|c|c|}
\hline /vespa / & $*+$ ant $] \sigma$ & ${ }_{\mathrm{v}}^{*} \mathrm{e} \int$ & Ident $_{\text {[post] }}$ & Ident $_{\text {[ant] }}$ & ${ }_{\mathrm{N}}^{*} \mathrm{e}$ \\
\hline CPF [vespa] & $*$ & & & & \\
\hline$\Leftrightarrow$ a.[veJp] & & & & $*$ & $*$ \\
\hline $\mathrm{b} \cdot\left[\mathrm{ve} \int \mathrm{pe}\right]$ & & & * & * & \\
\hline
\end{tabular}

No tableau acima, o candidato completamente fiel (identificado como $\mathrm{CPF}$ ) está destacado. Vemos, portanto, que o candidato opaco, a, vence a disputa com o candidato transparente, porque a restrição de marcação *e $\int$, na versão velha, não é violada por a.

Na versão clássica da TO, aquela que não admite que as restrições de marcação tenham acesso ao input, o ranking *e $\int>\operatorname{Ident}_{\text {[post] }}$ não permitia que [ve $\int \mathrm{pa}$ ] fosse escolhido. Por outro lado, o ranking contrário Ident ${ }_{[\text {post }]}>>$ *e $\int$ não permitiria explicar como ocorre a centralização em "fecho". Agora, o ranking ${ }_{\mathrm{V}}{ }^{*} \iint>$ Ident $_{[\text {post] }}>>{ }_{\mathrm{N}}{ }^{\mathrm{e}} \int$ permite explicar ambas. 
(18)

\begin{tabular}{|c|c|c|c|c|c|}
\hline$/ \mathrm{fe} \int \mathrm{o} /$ & $*+$ ant $] \sigma$ & $\mathrm{v}^{*} \mathrm{e} \int$ & Ident $_{\text {[post] }}$ & Ident $_{\text {[ant] }}$ & $\mathrm{N}^{* \mathrm{e} J}$ \\
\hline CPF [fe $\left.\int o\right]$ & & $*$ & & & \\
\hline a. $\left[\mathrm{fe} \int \mathrm{u}\right]$ & & $*$ & & & \\
\hline$\&$ b. [feu] & & & $*$ & & \\
\hline
\end{tabular}

A proposta modifica, na verdade, uma das propriedades que distinguiam as restrições de marcação de restrições de outros tipos. Para as restrições de fidelidade, a noção de correspondência (por exemplo, entre segmentos de input e output) é fundamental; ela também é importante para as restrições de alinhamento. As restrições de marcação - até esta proposta - não necessitavam da noção de correspondência. A partir de agora, as restrições de marcação podem e devem incorporar essa noção, caso contrário, não seria possível estabelecer comparações com o CPF. ${ }^{9}$

Através da estratégia de desdobramento das restrições de marcação, a Teoria da Otimidade pode dar conta da opacidade do tipo contra-alimentadora, em que há subaplicação de processo, sem abrir mão do paralelismo, mas ao custo de alguns acréscimos na engenharia de avaliação dos candidatos. O que a Marcação Comparativa tem em comum com outras propostas, como a Teoria da Simpatia, é a existência de um candidato perdedor com o qual os demais candidatos são comparados e que é diretamente responsável pela escolha do candidato ótimo. Esse candidato perdedor é o simpático na Teoria da Simpatia e o candidato plenamente fiel, na Teoria da Marcação Comparativa.

A Teoria da Marcação Comparativa parece intuitivamente mais simples do que a Teoria da Simpatia. Em princípio, o candidato plenamente fiel será sempre o mesmo e não depende crucialmente do ranking. A estratégia do desdobramento das restrições de marcação também parece menos estipulativa do que as escolhas, um tanto ad hoc, da restrição simpática e da restrição seletora.

Entretanto, há três aspectos em que a proposta parece não se sair tão bem quanto outras alternativas. Em primeiro lugar, a duplicação automática de todas as restrições de marcação aumenta em muito o número de restrições no componente CON, o que faz com que a teoria se torne mais indeterminada. Possivelmente não haverá evidência para todas as novas restrições de marcação criadas pela duplicação. Em segundo lugar, a proposta não se sai bem se - diante do postulado da Riqueza da Base - levarmos em consideração a possibilidade de inputs alternativos. Em terceiro lugar, a proposta faz determinadas predições 
a respeito das possibilidades de interação com outros processos, as quais não parecem se confirmar empiricamente. Na próxima seção, analisamos com mais detalhe esses dois aspectos.

\section{A Marcação Comparativa desafiada}

Quanto ao primeiro dos problemas, este já tinha sido identificado anteriormente para a Teoria da Simpatia, no que se refere ao tratamento de fenômenos opacos alofônicos (ITÔ; MESTER, 2003). Qual é esse problema? No caso da análise exposta, é o seguinte: a centralização da vogal, que resulta no mapeamento input/output /e/ $\rightarrow[\mathfrak{e}]$, é um processo alofônico. A centralização cria um segmento não-presente no inventário fonológico e não há contraste fonológico entre [e] e [e]; no contexto antes de segmento palatal, somente encontramos [e] e, nos demais ambientes, encontramos [e] ou outros alofones. ${ }^{10}$ Pela TO, as generalizações lingüísticas são dadas pela gramática e não podem ser obtidas pelo controle do que está no input. Isto implica dizer que tanto /e/ pode mapear a [e] quanto / $/ \mathfrak{e} /$ pode mapear a [e], ou seja, temos de verificar se a análise também funcionaria quando o segmento for o complemento no input. Para fins da exposição desse problema, é necessário considerar ainda uma restrição, a marcação *e, que proíbe vogais [-baixo, -arredondado, +posterior]. Sabemos que a vogal [e] tem distribuição restrita no português europeu, o que nos motiva a postular uma restrição que proíbe este tipo de vogal. Como há uma situação de distribuição complementar entre [e] e [e], consideramos que o seguinte esquema dê conta dos dados da língua: Restrição de marcação contextual $>>$ Restrição de marcação livre de contexto $>>$ Fidelidade (conforme Kager, 1999, cap.1), isto é ${ }_{\mathrm{v}} * \int>>* \mathrm{e}>>$ Ident $_{\text {[post] }}$

Se for assim, a análise dá certo no caso de "vespa":

(19)

\begin{tabular}{|c|l|l|l|l|l|l|}
\hline$/$ vespa / & $*+a n t] \sigma$ & $\mathrm{v}^{*} \mathrm{e} \int$ & $* \mathrm{e}$ & Ident $_{\text {[post] }}$ & Ident $_{\text {[ant] }}$ & ${ }_{\mathrm{N}}^{*} \int$ \\
\hline CPF [vespa] & $*$ & & $*$ & & & \\
\hline a. $\left[\mathrm{ve} \int \mathrm{p}\right]$ & & & & $*$ & $*$ & $*$ \\
\hline b. $\left[\mathrm{ve} \int \mathrm{pe}\right]$ & & & & & $*$ & \\
\hline
\end{tabular}

Mas não dá certo no caso de "facho". 
(20)

\begin{tabular}{|c|c|c|c|c|c|c|}
\hline$/ \mathrm{fe} \int \mathrm{o} /$ & $*+$ ant $] \sigma$ & $\mathrm{v}^{*} \mathrm{e} \int$ & $* p$ & Ident $_{\text {[post] }}$ & Ident $_{\text {[ant] }}$ & ${ }_{\mathrm{N}}^{*} \mathrm{eJ}$ \\
\hline $\mathrm{CPF}\left[\mathrm{fe} \int \mathrm{o}\right]$ & & & & & & - \\
\hline (:) a.[fe $\left.\int u\right]$ & & & & $*$ & & * \\
\hline b. $\left[\mathrm{fe} \int \mathrm{u}\right]$ & & & * & & & - \\
\hline
\end{tabular}

Esse problema é identificado por McCarthy (2002b). ${ }^{11}$

Quanto ao segundo problema, McCarthy (2002a), ao confrontar a Marcação Comparativa com a TO estratal, mostra que a primeira é uma teoria mais restrita do que a segunda (o que é desejável); entretanto, o autor alerta que provavelmente ela seja restrita demais.Uma das diferenças em termos de predições admitidas é com relação ao fato de que a marcação comparativa trata a opacidade contra-alimentadora como uma propriedade de uma gramática como um todo e não como propriedade de processos específicos. De acordo com o ranking ${ }_{\mathrm{V}} \mathrm{M} \gg \mathrm{F} \gg{ }_{\mathrm{N}} \mathrm{M}$, a fidelidade não será violada para eliminar violações novas, independentemente do que as origina. Assim, se vários processos diferentes produzem estruturas violadoras de $\mathrm{M}$, então todos eles estarão numa relação contra-alimentadora em relação ao processo definido pelo ranking ${ }_{\mathrm{v}} \mathrm{M} \gg \mathrm{F}$.

Nas teorias derivacionais, é possível ordenar as regras A, B e C de tal forma que A alimente B, a qual é contra-alimentada por C. Esse tipo de interação também pode ser obtido através da TO estratal (desde que seja possível distinguir dois níveis no componente lexical e que o processo opaco possa ser atribuído ao segundo nível e o processo alimentador possa ser atribuído ao primeiro).

No entanto, de acordo com a marcação comparativa, esse tipo de comportamento não deveria ser possível. Pelo ranking $\mathrm{F}>{ }_{\mathrm{N}} \mathrm{M}$, novas instâncias de M não podem ser resolvidas. Segundo McCarthy (2002a, p.43-44) "In general, comparative markedness predicts that, if a process is counterfed by at least one process, then it can never be fed by any other process in the same language."

Vejamos como isso se traduz no nosso exemplo. O mapeamento/e/ $\rightarrow[\mathrm{e}]$ viola fidelidade. Se a restrição de marcação é contra marcação antiga, , *e $\int$, e v ${ }^{*} \int$ domina fidelidade, então terá de ser atestado o mapeamento /e/ $\rightarrow[\mathrm{e}]$. Entretanto, se fidelidade domina ${ }_{\mathrm{N}}{ }^{*} \int$, nenhuma violação nova desencadeará $\mathrm{o}$ mapeamento infiel /e/ $\rightarrow[\mathrm{e}]$, ou seja, o ranking ${ }_{\mathrm{V}}{ }^{*} \mathrm{e} \int>>* / \mathrm{e} / \rightarrow[\mathrm{e}]>>_{\mathrm{N}}{ }^{*} \mathrm{e} \int$ prevê que nenhum processo alimente a centralização na língua.

Conforme se pode verificar em Vigário (2003, p. 82), há outros processos que contra-alimentam a centralização: 
- Harmonia vocálica verbal

$$
/ \mathrm{m} \varepsilon \int \mathrm{o} / \rightarrow \operatorname{me} \int \mathrm{o} \rightarrow{ }^{*} \mathrm{me} \int \mathrm{o}(\text { mexo })
$$

- Inserção opcional de glide

$$
/ \text { leeN/ } \rightarrow \text { lejje } \rightarrow \text { *lejje }(\text { lêem })
$$

- Incorporação de enclíticos

$$
\operatorname{de}+\kappa \rightarrow \operatorname{de} \kappa \mathrm{e} \rightarrow{ }^{*} \operatorname{de} \kappa \mathrm{e}(\text { dê-lha })
$$

Em todos esses processos, produzem-se as condições necessárias para a aplicação da regra de centralização. No primeiro caso, a vogal média-baixa torna-se média-alta, isto é, cria-se a vogal-alvo para a centralização. Nos outros dois exemplos, a modificação ocorre no contexto, de forma a criar o segmento palatal, gatilho da regra. O que os exemplos têm em comum é que a centralização não se aplicou, ou seja, o processo que produz o glide contra-alimentou a centralização. Nesse sentido, todos parecem trazer sustentação à predição da Marcação Comparativa.

No entanto, contrariando-a, há outros processos que alimentam a centralização (conforme MATEUS; D’ANDRADE, 2000).

- $\quad$ Assimilação de VT (MATEUS; D’ANDRADE, p. 78)

$$
/ \text { fal }+\mathrm{a}+\mathrm{i} / \rightarrow \text { falej } \rightarrow \text { falej (falei) }
$$

- Glidificação da lateral (MATEUS; D’ANDRADE, p. 71-72)

$$
\text { /fasil+s/ } \rightarrow \text { fasejs } \rightarrow \text { fasej } \int \text { (fáceis) }
$$

- Inserção de glide para desfazer ditongo (MATEUS; D'ANDRADE, p. 80)

$$
/ \text { pase }+\mathrm{o} / \rightarrow \text { pasejo } \rightarrow \text { peseju (passeio) }
$$

/are $+\mathrm{a} / \rightarrow$ areja $\rightarrow$ areje (areia)

Em todos os processos acima, produz-se um glide derivado, situação que cria contexto para a aplicação da regra de centralização. O que os exemplos mostram é que a centralização se aplicou, ou seja, o processo que produz o glide alimentou a centralização.

Além desses, podemos considerar também casos como el[ [ $]$ jo (elejo) e prot[[] jo (protejo) em que, conforme Vigário (2003), a harmonia vocálica verbal se aplica e a vogal elevada pela regra sofre, em seguida, a centralização. ${ }^{12}$

Os processos apresentados têm em comum o fato de não serem puramente fonológicos, pois todos eles têm aplicação restrita a determinados 
contextos morfológicos. Isso poderia nos levar a pensar que não sejam mais produtivos e que, portanto, não se configuram como reais contra-exemplos à predição. Entretanto, há também um fenômeno que não se enquadra nesta categoria e que também alimenta a centralização.

- Inserção de glide em sequiência VN (MATEUS; D’ANDRADE, 2000, p. 133)

$$
/ \text { viazeN/ } \rightarrow \text { viazêj } \rightarrow \text { viaz } \widetilde{j} \text { j (viagem) }
$$

Como reconhece McCarthy (2002a, p.43), a Marcação Comparativa provavelmente é uma teoria restrita demais para explicar a interação opaca. Pelos dados aqui analisados, parece ser mesmo demasiado restrita, porque, como vimos, a predição sobre a interação do processo de centralização com outros processos não se confirma.

\section{Conclusões}

A opacidade é um tema fascinante e há ainda muitas questões a serem exploradas. As diferentes soluções propostas recentemente têm de ser cotejadas com processos opacos das línguas para verificarmos sua real aplicabilidade na resolução dessa opacidade. A discussão da abordagem pela Marcação Comparativa mostrou-nos algumas de suas limitações. Em contrapartida, mostrou-nos também algumas indagações novas a respeito do funcionamento de processos como o da centralização da vogal /e/, colocando um foco diferente, novo, a respeito desses processos já conhecidos.

No que concerne às limitações da Marcação Comparativa como abordagem da opacidade, vimos que o tratamento de processos opacos com características alofônicas demanda desconsiderar a questão da Riqueza da Base. Além disso, referente à interação de múltiplos processos, a Marcação Comparativa prevê que nenhum fenômeno alimente a centralização da vogal no português europeu, já que nenhuma violação nova a *e justifica uma violação sucessiva da restrição de fidelidade que milita contra o mapeamento /e/ $\rightarrow[\mathrm{e}]$. Essa predição não se confirma, pois também encontramos fenômenos que alimentam a centralização. Os dados do PE aqui analisados confirmam, portanto, que a teoria é excessivamente restrita, porque exclui de explicação casos efetivamente observados na língua.

Por outro lado, a discussão sobre a interação opaca entre fenômenos com alofonia bem como a discussão sobre a interação de um fenômeno opaco com diversos outros fenômenos exemplificam indagações relevantes levantadas pela 
teoria. Em abordagens derivacionais, esse tipo de questão, importante para entender a natureza dos processos e da sua interação com outros processos, sequer era cogitada. Pela dificuldade que a TO enfrenta com a análise da interação opaca entre processos, damo-nos conta da real complexidade que essas interações representam, o que resulta, no mínimo, em descrições mais completas da fonologia das línguas, se não em tipologias mais apropriadas dos diferentes tipos de opacidade.

\section{Notas}

${ }^{1}$ Além disso, Vigário considera que a regra não precisa fazer referência à vogal tônica (VIGÁRIO, 2003, p. 81), desde que seja garantida a aplicação anterior da regra de redução vocálica.

${ }^{2}$ Vigário (2003, p. 80) considera que a especificação da fricativa ocorra ainda no léxico, razão por que recorre à condição de heterossilabicidade para explicar a não interação entre palatalização e centralização. Mencionando Morales-Front and Holt (1997), diz que a fricativa pós-vocálica teria de estar especificada como palatal no léxico para explicar a alternância entre um segmento nasal ou lateral e um glide palatal, em formas como animal/animais, pão/pães.

${ }^{3}$ A regra de palatalização é formalizada em Mateus e d'Andrade (2000, p. 37) como regra de preenchimento de traço [-anterior] a qual precede a regra default de preenchimento de [+anterior], ou seja, a fricativa pós-vocálica é subespecificada até o pós-léxico, quando recebe sua especificação conforme o contexto ou por default. Com essa formulação, há mais um argumento para não estipular a condição heterossilábica na regra de centralização. Bastaria estabelecer que o preenchimento de [-anterior] seja realizado no pós-léxico, quando a centralização já não está mais ativa, para que a regra não tenha aplicação nos casos de [ve $\left.\int \mathrm{pe}\right]$. Entretanto, para fins da presente discussão, consideraremos que a fricativa seja especificada subjacentemente como [+anterior]. Essa suposição será reavaliada mais adiante (v. nota 10). Outra questão que concerne à fricativa é a sua realização como [+vozeada] ou [-vozeada], que não será abordada aqui.

${ }^{4}$ Considerando-se apenas a primeira parte da definição, isto é, 'generalizações que não são detectáveis (i.e. válidas) na superfície', poderíamos incluir aqui muitos outros fenômenos como casos de 'opacidade'; por exemplo, casos em que a derivação morfológica obscureceu um contexto de aplicação de uma regra, ou casos em que fenômenos como reduplicação ou truncamento criam relações de correspondência entre duas formas, nas quais apenas uma delas possui contexto para aplicação de uma regra. Em conformidade com o emprego que McCarthy (2002b) faz do termo, não estenderemos a noção de opacidade para esses casos. 
${ }^{5}$ As restrições de fidelidade podem referir-se também ao input, mas nenhuma restrição refere-se exclusivamente ao input.

${ }^{6}$ A restrição tal qual vem formulada é bastante improvável. Uma restrição não deve pressupor o processo utilizado para remover sua violação, pois, nesse caso, ela passa a ser uma regra. A restrição em pauta parece considerar que a única solução à violação seja a mudança de /e/ para [e]. Para o momento, entretanto, não temos uma formulação mais adequada para ela.

${ }^{7}$ Entendemos que a restrição tanto pode ter como efeito mudança de traço (desde que o input esteja especificado como [+anterior]) quanto como inserção de traço [-anterior] (se o input for não-especificado para esse traço).

${ }^{8}$ Como vimos anteriormente, Mateus e d'Andrade (2000) consideram que a fricativa pós-vocálica seja subespecificada na subjacência quanto a ponto de articulação. Para fins da análise por restrições aqui apresentada, utilizamos a idéia de que a fricativa pós-vocálica esteja especificada no input como [+anterior].

${ }^{9}$ Surge daí uma questão: se as restrições de marcação incorporam a noção de correspondência, que garantias temos de que essa correspondência seja limitada a outro output? De que forma a TO poderá impedir que novas propostas permitam correspondência também ao input?

${ }^{10}$ A situação é, na verdade, um pouco mais complexa, já que encontramos [e]s que são alofones de /a/. Entretanto, essa observação não invalida o que estamos argumentando aqui.

${ }^{11}$ Entretanto, o fenômeno tratado apresenta uma complexidade adicional. Conforme McCarthy (2006b), quando houver uma situação em que /B/ e /C/ mapeiam ambos a [B] (como no caso da neutralização entre oclusivas vozeadas e desvozeadas em final de palavra, por exemplo, no alemão), podemos postular que tanto /B/ quanto $/ \mathrm{C} /$ estão no input, quando não houver alternância. McCarthy cita o caso da preposição 'und' "e", em que a realização desvozeada da oclusiva final não alterna com a realização vozeada, já que essa preposição não está sujeita a processos de derivação ou flexão. Segundo McCarthy, "If there are no alternations, (e.g. German und 'and.'), then the answer doesn't usually matter, so why worry about something that has no empirical consequences? (op.cit., p.2). A mesma posição poderia ser adotada aqui, no que se refere à questão de se $/ \mathrm{s} / \mathrm{ou} / \mathrm{S} /$ está no input. A palavra 'vespa' poderia teoricamente ter como input /ve $\int \mathrm{pa} /$. Entretanto, observe-se que o resultado, considerando uma restrição de marcação velha, como *e é é diferente se postularmos uma forma input como /ve $\int \mathrm{pa} /$. Então, fica evidente que postularmos uma forma ou outra no input tem consequiências empíricas para a análise. 
${ }^{12}$ De acordo com o que se pode depreender da explicação de Vigário, essa seria a situação normal, ao passo que os casos em que a centralização não se aplica à vogal derivada por harmonia vocálica, como mexo e rejo, seriam as exceções.

\section{Referências Bibliográficas}

ABAURRE, M. B.; PAGOTTO, E. G. Palatalização das oclusivas dentais no português do Brasil. In: ABAURRE, M.B.M.; RODRIGUES, A.C.S. (Ed.). Gramática do português falado VIII. Campinas: Editora da UNICAMP. 2002. p. 557-602.

BISOL, L. Palatalization and its variable restriction. In: MATTOS; BORTONI (Ed.). International Journal of the Sociology of Language, v. 89, p.107-124, 1991.

D’ANDRADE, E. Temas de Fonologia. Lisboa: Colibri, 1994.

ITÔ, J.; MESTER, A. Lexical and Postlexical Phonology in Optimality Theory: Evidence from Japanese. Manuscrito. 2003. Disponível em: <http://roa.rutgers.edu>. Acesso em: 08/11/2006.

LEE, S.-H. Formas de entrada e otimização do léxico. Ms., 2002.

KAGER, R. Optimality Theory. Cambridge: CUP, 1999.

KIPARSKY, P. Linguistic universals and linguistic change. In: BACH, E.; HARMS, R. Universals in linguistic theory. New York: Holt, Rinehart and Winston, 1968.

KIPARSKY, P. Historical Linguistics. In: DINGWALL(Ed.). A Survey of Linguistic Science. University of Maryland Press, 1971. p.577-642.

KIPARSKY, P. Opacity and cyclicity. The Linguistic Review, n. 17, p. 351-365, 2000.

KIRCHNER, R. Synchronic chain shifts in Optimality Theory. Linguistic Inquiry, v. 27, p. 341-349, 1996.

MATEUS, M. H. et alii. Fonética, fonologia e morfologia do português. Lisboa: Universidade Aberta, 1990.

MATEUS, M.; D'ANDRADE, E. The phonology of Portuguese. Oxford: Oxford University Press, 2000.

MCCARTHY, J. Sympathy and phonological opacity. Phonology, v.16, p.331-399, 1999.

McCARTHY, J. Comparative Markedness. Amherst: University of Massachusetts, 2002a. Disponível em: http://roa.rutgers.edu.

McCARTHY, J. A thematic Guide to Optimality Theory. Cambridge: Cambridge University Press, $2002 b$. 
McCARTHY, J. 2006a GEN, EVAL, and Phonological Opacity. Indiana University Bloomington. Phonology Fest 2006. Disponível em: <http://www.indiana.edu/ $\sim$ phono/page1/McCarthyHO.pdf > acesso em 08/11/2006

McCARTHY, J. (2006b) Handout: Inventories and distribution in OT. Linguistics 603. Amherst: University of Massachusetts. Disponível em: <http:// people.umass.edu/jjmccart/ling603/>. Acesso em: 08/11/2006.

RUBACH, J. Glide and glottal stop insertion in Slavic Languages: a DOT Analysis. Linguistic Inquiry, v.31, n.2, p. 271-317, 2000.

VIEIRA, M. J. B. As vogais médias postônicas: uma análise variacionista. In: BISOL, L; BRESCANCINI, C. (Org.). Fonologia e Variação: Recortes do português brasileiro. Porto Alegre: EDIPUCRS, 2002. p.127-159.

VIGÁRIO, M. The Prosodic Word in European Portuguese, Berlin \& New York: Mouton de Gruyter, Interface Explorations 6, 2003. 440 p. 